\title{
Probing gravitation with pulsars
}

\author{
Michael Kramer ${ }^{1,2}$ \\ ${ }^{1}$ MPI für Radioastronomy, Auf dem Hügel 69, 53121 Bonn, Germany \\ email: mkramer@mpifr.de \\ ${ }^{2}$ Jodrell Bank Centre for Astrophysics, University of Manchester \\ Oxford Road, Manchester M13 9PL, UK \\ email: michael.kramer@manchester.ac.uk
}

\begin{abstract}
Radio pulsars are fascinating and extremely useful objects. Despite our on-going difficulties in understanding the details of their emission physics, they can be used as precise cosmic clocks in a wide-range of experiments - in particular for probing gravitational physics. While the reader should consult the contributions to these proceedings to learn more about this exciting field of discovering, exploiting and understanding pulsars, we will concentrate here on on the usage of pulsars as gravity labs.
\end{abstract}

Keywords. gravitation, techniques:radio astronomy, stars:neutron, pulsars:general

\section{Introduction}

We are less than three years away from celebrating the centenary of Einstein's theory of general relativity (GR). Nearly a hundred years later, efforts in testing GR and its concepts are still being made by many colleagues around the world, using many different approaches. To date GR has passed all experimental and observational tests with flying colours, but in light of recent progress in observational cosmology in particular, the question of as to whether alternative theories of gravity need to be considered is as topical as ever.

Many experiments are designed to achieve ever more stringent tests by either increasing the precision of the tests or by testing different, new aspects. Some of the most stringent tests are obtained by satellite experiments in the solar system, providing exciting limits on the validity of GR and alternative theories of gravity. However, solar-system experiments are made in the gravitational weak-field regime, while deviations from GR may appear only in strong gravitational fields.

We are all very much looking forward to the first direct detection of gravitational waves with ground-based (and hopefully, eventually, space-based) detectors, which not only open a completely new window to the Universe but which will also provide superb tests of GR. Meanwhile, it happens that nature provides us with an almost perfect laboratory to test the strong-field regime - in the form of binary radio pulsars.

While, strictly speaking, the binary pulsars move in the weak gravitational field of a companion, they do provide precision tests of the strong-field regime. This becomes clear when considering that the majority of alternative theories predicts strong self-field effects which would clearly affect the pulsars' orbital motion. By "simply" measuring the arrival time of pulsars moving in the curved space-time of their companion, we can locate their position in their orbit with an uncertainty as little as 20-50 m! Hence, tracing their fall in a gravitational potential, we can search for tiny deviations from GR, providing us with unique precision strong-field tests of gravity. 


\section{Simple and clean experiments}

The use of pulsars for tests of gravitational physics with "pulsar timing" is a clean and conceptually simple experiment. By simply measuring the exact arrival time of pulses at our telescope on Earth, we do a ranging experiment that is vastly superior in precision than a simple measurement of Doppler-shifts in the pulse period. In fact, the pulsed nature of our signal that links tightly and directly to the rotation of the neutron star, allows us to count every single rotation of a neutron star (see contribution by Scott Ransom for details). In this experiment we can consider the pulsar as a test mass that has a precision clock attached to it and which allows us to follow the movement of the test mass in the curved space-time of the companion. As a result, a wide range of relativistic effects can be observed, identified and studied. These include so far:

- Precession of periastron

- Gravitational redshift

- Shapiro delay due to curved space-time

- Gravitational wave emission

- Geodetic precession, relativistic spin-orbit coupling

- Speed of gravity

But we can also convert our observations in the tests of concepts and principles deeply embedded in theoretical frameworks, such as

- Strong Equivalence Principle (grav. Stark effect),

- Lorentz invariance of gravitational interaction,

- Non-existence of preferred frames,

- Conservation of total momentum,

- Non-variation of gravitational constant,

which also leads to stringent limits on alternatives theories of gravity, e.g. tensor-scalar theories, Tensor-Vector-Scalar (TeVeS) theories.

\subsection{Pick your laboratory!}

The various effects or concepts to be tested require sometimes a rather different type of laboratory. For instance, to test the violation of the Strong-Equivalence-Principle, one would like to use a binary system that consists of different types of masses (i.e. with different gravitational self-energy), rather than a system made of very similar bodies. Fortunately, nature has been kind.

At the moment, we know about 2000 pulsars, with about $10 \%$ of these in binary systems. The shortest orbital period is about $90 \mathrm{~min}$ while the longest period is $5.3 \mathrm{yr}$ (e.g. Lorimer \& Kramer 2005). We find different types of components, i.e. main-sequence stars, white dwarfs (WD), neutron stars (NS) and even planets. Unfortunately, despite past and on-going efforts, we have not yet found a pulsar about a stellar black hole companion or about the supermassive black hole in the centre of our Galaxy (Liu et al. 2012). Double neutron star systems are rare but usually produce the largest observable relativistic effects in their orbital motion and, as we will see, produce the best tests of general relativity for strongly self-gravitating bodies. In comparison, pulsar - white dwarf systems are much more common. Indeed, most pulsar companions are white dwarfs, with a wide range of orbital periods, ranging from hours to days, weeks and months. Still, many of them can be used for tests of gravitational theories where we utilize the fact that white dwarfs and neutron stars differ very significantly in their structure and, consequently, self-energies. 
Table 1. Examples of precision measurements using pulsar timing as a demonstration what is possible today. The digit in bracket indicates the uncertainty in the last digit of each value. References are cited. More details on gravitational wave detection with pulsar timing arrays can be found in the contributions by Hobbs, Liu, Shannon and others.

\begin{tabular}{|c|c|c|}
\hline \\
\hline $\begin{array}{l}\text { Masses: } \\
\text { Masses of neutron stars: }\end{array}$ & $m_{1}=1.4398(2) M_{\odot}$ & (Weisberg et al. 2010) \\
\hline Mass of WD companion: & $0.207(2) M_{\odot}$ & (Hotan et al. 2006) \\
\hline Mass of millisecond pulsar: & $1.67(2) M_{\odot}$ & (Freire et al. 2010) \\
\hline Main sequence star companion: & $1.029(8) M_{\odot}$ & (Freire et al. 2010) \\
\hline Mass of Jupiter and moons: & $9.547921(2) \times 10^{-4} M_{\odot}$ & (Champion et al. 2010) \\
\hline $\begin{array}{l}\text { Spin parameters: } \\
\text { Period: }\end{array}$ & $5.757451924362137(2) \mathrm{ms}$ & (Verbiest et al. 2008) \\
\hline Orbital parameters: & & \\
\hline Period: & $0.102251562479(8)$ day & (Kramer et al. in prep.) \\
\hline Eccentricity: & $3.5(1.1) \times 10^{-7}$ & (Freire et al. in 2012) \\
\hline Astrometry: & & \\
\hline Distance: & $157(1) \mathrm{pc}$ & (Verbiest et al. 2008) \\
\hline Proper motion: & $140.915(1)$ mas yr$^{-1}$ & (Verbiest et al. 2008) \\
\hline Tests of general relativity: & & \\
\hline Periastron advance: & $4.226598(5) \operatorname{deg} \mathrm{yr}^{-1}$ & (Weisberg et al. 2010) \\
\hline Shrinkage due to GW emission: & $7.152(8) \mathrm{mm} /$ day & (Kramer et al. in prep) \\
\hline GR validity (obs/exp): & $1.0000(5)$ & (Kramer et al. in prep.) \\
\hline Constancy of grav. Constant, $\dot{G} / G$ : & $9(12) \times 10^{-13} \mathrm{yr}^{-1}$ & (Zhu et al. in prep) \\
\hline $\begin{array}{l}\text { Gravitational wave detection: } \\
\text { Change in relative distance: }\end{array}$ & $100 \mathrm{~m} / 1$ lightyear & (EPTA, NANOGrav, PPTA) \\
\hline
\end{tabular}

\subsection{Not only clean, but also very precise}

We follow the pulsars in their orbit by registering the variation in arrival time as they move around the system's centre of mass. Using pulsar timing techniques, we make extremely precise measurements that allow us to probe gravitation with exquisite accuracy. Table 1 gives an idea about the precision that we already achieve today. With future telescopes like the "Square Kilometre Array", the precision will even be enhanced by at least two orders of magnitudes and should, for instance, allow us to find a pular orbiting SGR $\mathrm{A}^{*}$, which would provide the mass of the central black hole to a precision of an amazing $1 M_{\odot}$ ! It would also allow us to measure the spin of the black hole with a precision of $10^{-4}$ to $10^{-3}$ (enabling tests of the "cosmic censorship conjecture") and the quadrupole moment with a precision to $10^{-4}$ to $10^{-3}$ (thus enabling tests of the "no-hair theorem"). See Liu et al. (2012) and the contribution by Wex et al. for more details.

\section{The first laboratory}

The first binary pulsar to ever be discovered happend to be a rare double neutron star system. It was discovered by Russel Hulse and Joe Taylor in 1974 (Hulse \& Taylor 1975). The pulsar, B1913+16, has a period of $59 \mathrm{~ms}$ and is in eccentric $(e=0.61)$ orbit around a unseen companion with an orbital period of less than 8 hours. It became soon clear that the pulsar does not follow the movement expected from a simple Keplerian description of the binary orbit, but that it shows the impact of relativistic effects. In order to describe the relativistic effects in a theory-independent fashion, one introduces so-called 
"Post-Keplerian" (PK) parameters that are included in a timing model to accurately describe the measured pulse times-of-arrival (see e.g. contribution by David Nice for more details).

For the Hulse-Taylor pulsar, it was soon measured that the system showed a relativistic advance of its periastron, comparable to what is seen in the solar system for Mercury, albeit with a much larger amplitude of $\dot{\omega}=4.226598 \pm 0.000005 \mathrm{deg} / \mathrm{yr}$ (Weisberg et al. 2010). GR predicts a value for the periastron advance that depends on the Keplerian parameters and the masses of the pulsar and its companion:

$$
\dot{\omega}=3 T_{\odot}^{2 / 3}\left(\frac{P_{\mathrm{b}}}{2 \pi}\right)^{-5 / 3} \frac{1}{1-e^{2}}\left(m_{\mathrm{p}}+m_{\mathrm{c}}\right)^{2 / 3} .
$$

Here, $T_{\odot}$ is a constant, $P_{\mathrm{b}}$ the orbital period, $e$ the eccentricity, and $m_{\mathrm{p}}$ and $m_{\mathrm{c}}$ the masses of the pulsar and its companion. See Lorimer \& Kramer (2005) for further details.

The Hulse-Taylor pulsars also shows the effects of gravitational redshift (including a contribution from a second-order Doppler effect) as the pulsar moves in its elliptical orbit at varying distances from the companion and with varying speeds. The result is a variation in the clock rate of with an amplitude of $\gamma=4.2992 \pm 0.0008 \mathrm{~ms}$ (Weisberg et al. 2010). In GR, the observed value is related to the Keplerian parameters and the masses as

$$
\gamma=T_{\odot}^{2 / 3}\left(\frac{P_{\mathrm{b}}}{2 \pi}\right)^{1 / 3} e \frac{m_{\mathrm{c}}\left(m_{\mathrm{p}}+2 m_{\mathrm{c}}\right)}{\left(m_{\mathrm{p}}+m_{\mathrm{c}}\right)^{4 / 3}} .
$$

We can now combine these measurements. We have two equations with a measured lefthand side. On the right-hand side, we measured everything apart from two unknown masses. We solve for those and obtain, $m_{\mathrm{p}}=1.4398 \pm 0.0002 M_{\odot}$ and $m_{\mathrm{c}}=1.3886 \pm$ $0.0002 M_{\odot}$ (Weisberg et al. 2010). These masses are correct if GR is the right theory of gravity. If that is indeed the case, we can make use of the fact that (for point masses with negligible spin contributions), the PK parameters in each theory should only be functions of the a priori unknown masses of pulsar and companion, $m_{\mathrm{p}}$ and $m_{\mathrm{c}}$, and the easily measurable Keplerian parameters (Damour \& Taylor 1992) $\dagger$. With the two masses now being determined using GR, we can compare any observed value of a third PK parameter with the predicted value. A third such parameter is the observed decay of the orbit which can be explained fully by the emission of gravitational waves. And indeed, using the derived masses, and the prediction of general relativity, i.e.

$$
\dot{P}_{\mathrm{b}}=-\frac{192 \pi}{5} T_{\odot}^{5 / 3}\left(\frac{P_{\mathrm{b}}}{2 \pi}\right)^{-5 / 3} \frac{\left(1+\frac{73}{24} e^{2}+\frac{37}{96} e^{4}\right)}{\left(1-e^{2}\right)^{7 / 2}} \frac{m_{\mathrm{p}} m_{\mathrm{c}}}{\left(m_{\mathrm{p}}+m_{\mathrm{c}}\right)^{1 / 3}}
$$

one finds an agreement with the observed value of $\dot{P}_{\mathrm{b}}^{\text {obs }}=(2.423 \pm 0.001) \times 10^{-12}$ (Weisberg et al. 2010) - however, only if a correction for a relative acceleration between the pulsar and the solar system barycentre is taken into account. As the pulsar is located about $7 \mathrm{kpc}$ away from Earth, it experiences a different acceleration in the Galactic gravitational potential than the solar system (see e.g. Lorimer \& Kramer 2005). The precision of our knowledge to correct for this effect eventually limits our ability to compare the GR prediction to the observed value. Nevertheless, the agreement of observations and prediction, today within a $0.2 \%$ (systematic) uncertainty (Weisberg et al. 2010), represented the first evidence for the existence of gravitational waves. Today we know many more binary pulsars where we can detect gravitational wave emission. In one particular

$\dagger$ For alternative theories of gravity this statement may only be true for a given equation-ofstate. 
case, the measurement uncertainties are not only more precise, but also the systematic uncertainties are much smaller, as the system is much more nearby. This system is the Double Pulsar.

\section{The Double Pulsar}

The Double Pulsar was discovered in 2003 (Burgay et al. 2003, Lyne et al. 2004). It does not only show larger relativistic effects and is much closer to Earth (about $1 \mathrm{kpc}$ ) than the Hulse-Taylor pulsar, allowing us to largely neglect the relative acceleration effects, but the defining unique property of the system is that it does not consist of one active pulsar and its unseen companion, but that it harbours two active radio pulsars.

One pulsar is mildly recycled with a period of $22 \mathrm{~ms}$ (named "A"), while the other pulsar is young with a period of $2.8 \mathrm{~s}$ (named " $\mathrm{B}$ "). Both orbit the common centre of mass in only 147-min with orbital velocities of 1 Million $\mathrm{km}$ per hour. Being also mildly eccentric $(e=0.09)$, the system is an ideal laboratory to study gravitational physics and fundamental physics in general. A detailed account of the exploitation for gravitational physics has been given, for instance, by Kramer et al. (2006), Kramer \& Stairs (2008) and Kramer \& Wex (2009). An update on those results is in preparation (Kramer et al., in prep.), with the largest improvement undoubtedly given by a large increase in precision when measuring the orbital decay. Not even ten years after the discovery of the system, the Double Pulsar provides the best test for the accuracy of the gravitational quadrupole emission prediction by GR far below the $0.1 \%$ level.

In order to perform this test, we first determine the mass ratio of pulsar $\mathrm{A}$ and $\mathrm{B}$ from their relative sizes of the orbit, i.e. $R=x_{B} / x_{A}=m_{A} / m_{B}=1.0714 \pm 0.0011$ (Kramer et al. 2006). Note that this value is theory-independent to the $1 \mathrm{PN}$ level (Damour \& Deruelle 1986). The most precise PK parameter that can be measured is a large orbital precession, i.e. $\dot{\omega}=16.8991 \pm 0.0001 \mathrm{deg} / \mathrm{yr}$. Using Eq. (3.1), this measured value and the mass ratio, we can determine the masses of the pulsars, assuming GR is correct, to be $m_{A}=(1.3381 \pm 0.0007) M_{\odot}$ and $m_{B}=(1.2489 \pm 0.0007) M_{\odot}$.

We can use these masses to compute the expected amplitude for the gravitational redshift, $\gamma$, if GR is correct. Comparing the result with the observed value of $\gamma=383.9 \pm$ $0.6 \mu \mathrm{s}$, we find that theory (GR) agrees with the observed value to a ratio of $1.000 \pm 0.002$, as a first of five tests of GR in the Double Pulsar.

The Double Pulsar also has the interesting feature that the orbit is seen nearly exactly edge-on. This leads to a 30-s long eclipse of pulsar A due to the blocking magnetosphere of B that we discuss further below, but it also leads to a "Shapiro delay": whenever the pulse needs to propagate through curved space-time, it takes a little longer than travelling through flat space-time. At superior conjunction, when the signal of pulsar A passes the surface of $B$ in only $20,000 \mathrm{~km}$ distance, the extra path length due to the curvature of space-time around B leads to an extra time delay of about $100 \mu \mathrm{s}$. The shape and amplitude of the corresponding Shapiro delay curve yield two PK parameters, $s$ and $r$, known as shape and range, allowing two further tests of GR. $s$ is measured to $s=\sin (i)=0.99975 \pm 0.00009$ and is in agreement with the GR prediction of

$$
s=T_{\odot}^{-1 / 3}\left(\frac{P_{\mathrm{b}}}{2 \pi}\right)^{-2 / 3} x \frac{\left(m_{A}+m_{B}\right)^{2 / 3}}{m_{B}},
$$

(where $x$ is the projected size of the semi-major axis measured in lt-s) within a ratio of $1.0000 \pm 0.0005$. It corresponds to an orbital inclination angle of $88.7 \pm 0.2 \mathrm{deg}$, which is indeed very close to $90 \mathrm{deg}$ as suggested by the eclipses. $r$ can be measured with much 
less precision and yields an agreement with GR's value given by

$$
r=T_{\odot} m_{B}
$$

to within a factor of $0.98 \pm 0.02$.

A fourth test is given by comparing an observed orbital decay of $107.79 \pm 0.11 \mathrm{~ns} /$ day to the GR prediction. Unlike the Hulse-Taylor pulsar, extrinsic effects are negligible and the values agree with each other without correction to within a ratio of $1.000 \pm 0.001$. This is already a better test for the existence of GW than possible with the Hulse-Taylor pulsar and will continue to improve with time. Indeed, at the time of writing the agreement has already surpassed the $0.1 \%$ level significantly (Kramer et al., in prep.).

\section{Relativistic spin-orbit coupling}

Apart from the Shapiro-delay, the impact of curved space-time is also immediately measurable by its effect on the orientation of the pulsar spin in a gyroscope experiment. This effect, known as geodetic precession or de Sitter precession represents the effect on a vector carried along with an orbiting body such that the vector points in a different direction from its starting point (relative to a distant observer) after a full orbit around the central object. Experimental verification has been achieved by precision tests in the solar system, e.g. by Lunar Laser Ranging (LLR) measurements, or recently by measurements with the Gravity Probe-B satellite mission (see Will 2006 for a review of experimental tests). However, these tests are done in the weak field conditions of the solar system, so that pulsars provide the only access to the strong-field regime.

In binary systems one can interpret the observations, depending on the reference frame, as a mixture of different contributions to relativistic spin-orbit interaction. One contribution comes from the motion of the first body around the centre of mass of the system (deSitter-Fokker precession), while the other comes from the dragging of the internal frame at the first body due to the translational motion of the companion (Börner et al. 1975). Hence, even though we loosely talk about geodetic precession, the result of the spin-orbit coupling for binary pulsar is more general, and hence we will call it relativistic spin-precession. The consequence of relativistic spin-precession is a precession of the pulsar spin about the total angular moment vector, changing the orientation of the pulsar relative to Earth.

Since the orbital angular momentum is much larger than the angular momentum of the pulsar, the orbital spin practically represents a fixed direction in space, defined by the orbital plane of the binary system. Therefore, if the spin vector of the pulsar is misaligned with the orbital spin, relativistic spin-precession leads to a change in viewing geometry, as the pulsar spin precesses about the total angular momentum vector. Consequently, as many of the observed pulsar properties are determined by the relative orientation of the pulsar axes towards the distant observer on Earth, we should expect a modulation in the measured pulse profile properties, namely its shape and polarisation characteristics (Damour \& Ruffini 1974). The precession rate is another PK parameter and given in GR by (e.g. Lorimer \& Kramer 2005)

$$
\Omega_{\mathrm{p}}=T_{\odot}^{2 / 3} \times\left(\frac{2 \pi}{P_{\mathrm{b}}}\right)^{5 / 3} \times \frac{m_{\mathrm{c}}\left(4 m_{\mathrm{p}}+3 m_{\mathrm{c}}\right)}{2\left(m_{\mathrm{p}}+m_{\mathrm{c}}\right)^{4 / 3}} \times \frac{1}{1-e^{2}}
$$

In order to see a measurable effect in any binary pulsar, $a$ ) the spin axis of the pulsar needs to be misaligned with the total angular momentum vector and $b$ ) the precession rate must be sufficiently large compared to the available observing time to detect a change in the 
emission properties. Table 2 lists the known Double Neutron Star Systems which typically show the largest degree of relativistic effects due to the often short eccentric binary orbits. However, the last entry in the table is PSR J1141-6545 which is a relativistic system with a white dwarf companion. Those pulsars that are marked with an asterisk have been identified as pulsars showing relativistic spin precession. Note that the top 5 out of 8 sources (with a known expected precession rate) indeed show the effect.

As the most relativistic binary system known to date, we expect a large amount of spin precession in the Double Pulsar system. Despite careful studies, profile changes for A have not been detected, suggesting that A's misalignment angle is rather small (e.g. Ferdman et al. 2012). In contrast, changes in the light curve and pulse shape on secular timescales (Burgay et al. 2005) reveal that this is not the case for B. In fact, B had been becoming progressively weaker and disappeared from our view in 2009 (Perera et al. 2010). Making the valid assumption that this disappearance is solely caused by relativistic spin precession, it will only be out of sight temporarily until it reappears later. Modelling suggests that, depending on the beam shape, this will occur in about 2035 but an earlier time cannot be excluded. The geometry that is derived from this modelling is consistent with the results from complementary observations of spin precession, visible via a rather unexpected effect described in the following.

The change on the orientation of $\mathrm{B}$ also changes the observed eclipse pattern in the Double Pulsar, where we can see periodic bursts of emission of A during the dark eclipse phases, with the period being the full- or half-period of B. As this pattern is caused by the rotation of B's blocking magnetospheric torus that allows light to pass B when the torus rotates to be seen from the side, the resulting pattern is determined by the three-dimensional orientation of the torus, which is centred on the precessing pulsar spin. Eclipse monitoring over the course of several years shows exactly the expected changes, allowing to determine the precession rate to $\Omega_{\mathrm{p}, \mathrm{B}}=4.77_{-0.65}^{+0.66} \mathrm{deg} / \mathrm{yr}$. This value is fully consistent with the value expected GR, providing a fifth test (Breton et al. 2008). This measurement also allows to test alternative theories of gravity and their prediction for relativistic spin-precession in strongly self-gravitating bodies for the first time (see Kramer \& Wex 2009 for details).

\section{Alternative theories}

Despite the successes of GR, a range of observational data have fuelled the continuous development of alternative theories of gravity. Such data include the apparent observation of "dark matter" or the cosmological results interpreted in the form of "inflation" and "dark energy". Confronting alternative theories with data also in other areas of the parameter space (away from the CMB or Galactic scales), requires that these theories are developed sufficiently in order to make predictions. A particular sensitive criterion is if the theory is able to make a statement about the existence and type of gravitational waves. Most theories cannot (yet), but a class of theories where this has been achieved is the class of tensor-scalar theories as discussed and demonstrated by Damour and EspositoFarèse in a series of works (e.g. Damour \& Esposito-Farèse 1996). For corresponding tests, the choice of a double neutron star system is not ideal, as the difference in scalar change, (that would be relevant, for instance, for the emission of gravitational dipole radiation) is small. The ideal laboratory would be a pulsar orbiting a black hole, as the black hole would have zero scalar charge and the difference would be maximised. The next best laboratory is a pulsar-white dwarf system. Indeed, such binary systems are able to provide constraints for alternative theories of gravity that are equally good or even better than solar system limits (Freire et al. 2012). 
Table 2. DNSs sorted according to the expected relativistic spin precession rate. Also included is PSR J1141-6545 which is in a relativistic orbit about a white dwarf companion. Pulsars marked with an asterisk have been identified of showing spin precession. For sources where no precession rate is listed, the companion mass could not be accurately measured yet, indicating however, that the precession rate is low.

\begin{tabular}{lrcccc}
\hline PSR & $P(\mathrm{~ms})$ & $P_{\mathrm{b}}(\mathrm{d})$ & $x(\mathrm{lt}-\mathrm{s})$ & $e$ & $\Omega_{\mathrm{p}}\left(\mathrm{deg} \mathrm{yr}^{-1}\right)$ \\
\hline J0737-3039A/B* & $22.7 / 2770$ & 0.10 & $1.42 / 1.51$ & 0.09 & $4.8 / 5.1$ \\
$\mathrm{~J}_{1906+0746 *}$ & 144.1 & 0.17 & 1.42 & 0.09 & 2.2 \\
B2127+11C* & 30.5 & 0.34 & 2.52 & 0.68 & 1.9 \\
B1913+16* & 59.0 & 0.33 & 2.34 & 0.62 & 1.2 \\
$\mathrm{~J} 1756-2251$ & 28.5 & 0.32 & 2.76 & 0.18 & 0.8 \\
B1534+12* & 37.9 & 0.42 & 3.73 & 0.27 & 0.5 \\
$\mathrm{~J} 1829+2456$ & 41.0 & 1.18 & 7.24 & 0.14 & 0.08 \\
$\mathrm{~J} 1518+4904$ & 40.9 & 8.64 & 20.0 & 0.25 & - \\
$\mathrm{J} 1753-2240$ & 95.1 & 13.63 & 18.1 & 0.30 & - \\
$\mathrm{J} 1811-1736$ & 104.2 & 18.8 & 34.8 & 0.83 & - \\
$\mathrm{J} 1141-6545^{*}$ & 394.0 & 0.20 & 1.89 & 0.17 & 1.4 \\
\hline
\end{tabular}

\section{Summary \& Conclusions}

A variety of experiments and observational data exist that allow us to test our understanding of gravity with increased precision. So far, general relativity has passed all tests with flying colours but the apparent existence of "Dark Energy" challenges this simple picture. It is clear that the observations of pulsars will continue to play an important part in testing general relativity and its alternatives. We expect to detect gravitational waves not only indirectly but also directly using pulsar observations (see corresponding contributions), and we have all reasons to believe that future searches will yield pulsars that can probe the space-time around black holes. Combined with the results of other experiments, namely the detection of gravitational waves with ground based detectors, we can expect a bright future for our understanding of gravity.

\section{References}

Börner G., Ehlers, J. \& Rudolph, E., 1975, A\&A, 44, 417

Breton R. P., et al., 2008, Science, 321, 104

Burgay M., et al., 2003, Nature, 426, 531

Burgay M., et al., 2005, ApJ, 624, L113

Damour T. \& Deruelle N., 1986, Ann. Inst. H. Poincaré (Physique Théorique), 44, 263

Damour T. \& Esposito-Farèse G., 1996, Phys. Rev. D, 54, 1474

Damour T. \& Ruffini R., 1974, Acad. des Sci. Paris Comptes Rendus Ser. Scie. Math., 279, 971

Damour T. \& Taylor J. H., 1992, Phys. Rev. D, 45, 1840

Ferdman R. D., et al., 2012, submitted

Freire P. C. C., et al., 2012, MNRAS, 423, 3328

Hulse R. A. \& Taylor J. H., 1975, ApJ, 195, L51

Kramer M. \& Stairs I. H., 2008, Ann. Rev. Astr. Ap., 46, 541

Kramer M. \& Wex N., 2009, Classical and Quantum Gravity, 26(7), 073001

Kramer M., et al., 2006, Science, 314, 97

Liu K., Wex N., Kramer M., Cordes J. M., \& Lazio T. J. W., 2012, ApJ, 747, 1

Lorimer D. R. \& Kramer M., 2005, Handbook of Pulsar Astronomy. Cambridge University Press Lyne A. G., et al., 2004, Science, 303, 1153

Perera B., et al., 2010, ApJ, 721, 1193

Weisberg J. M., Nice D. J. \& Taylor J. H., 2010, ApJ, 722, 1030

Will C., 2006, Living Reviews in Relativity, 9, 1 